\title{
DETERMINATION OF VIRULENCE FACTORS IN Haemophilus parasuis ISOLATES
}

\author{
V.I. PAVELKO, Yu.Yu. BABIN, A.V. SPRYGIN, O.V. PRUNTOVA \\ Federal Center for Animal Health Control, FGBU VNIIZZh, mkr. Yurievets, Vladimir, 600901 Russia, e-mail \\ vasily.pavelko@icloud.com, sprygin@arriah.ru; \\ Acknowledgements: \\ The authors thank N.V. Volozhantsev (State Research Center for Applied Microbiology and Biotechnology, \\ Obolensk) for providing isolates of Haemophilus parasuis. \\ Supported by Ministry of Agriculture of the Russian Federation \\ Received March 29, 2016 \\ doi: 10.15389/agrobiology.2016.4.491eng
}

\section{Abstract}

Glasser's disease is a bacterial infection typically characterized by fibrinous polyserositis, polyarthritis, meningitis, and occasionally acute pneumonia and septicemia. Haemophilus parasuis is the etiologic agent of Glasser's disease, leading to significant economic losses in swine industry. $H$. parasuis normally colonizes the upper respiratory tract of healthy pigs. Significant variation in virulence, serological and genetic properties has been shown across $H$. parasuis strains. However, stress, concomitant viral infections, or poor immune status allow $H$. parasuis to cause disease. The current literature contains a few studies that clarify differences in the genome between highly virulent and avirulent strains in the context of putative virulence factors. The objective of this study was to examine $H$. parasuis strains isolated on Russian pig farms during the 11-year study period (20002011) for the presence of putative virulence loci. We screened 6 strains and 23 field isolates for the presence of 10 virulence factors. Identification of NAD-dependent isolates was performed by biochemical analysis and species-specific real-time PCR. Putative virulence genes were amplified by PCR using different primer pairs. Genetic analysis confirmed that the virulence factors (vtaA, fhuA, hhdA, hhdB, nhaC, HAPS_0254, sclB7, sclB11 and phage_related) exist in different combinations. Out of 28 strains studied, the vtaA gene was found in all strains, sclB7 and $s c l B 11$ genes were present in 24 and 25 strains respectively, the cirA gene in 18 out of 28 , the fhuA gene in HAPS_0254, the hhdA gene in 15, the $h h d B$ gene in 14 , the nhaC gene in 12, the phage_related target in only 3 strains. Our findings identified 15 novel genotypes (LUB, Ural'skii-DEP, MB, SK-1DEP, SK3, Krasnodar, Botovo-2, Botovo-5, Botovo-7, IL2, AI4, V171, SH6, SW124, VN). The strains IL-1-DEP, KOMI DEP and isolate Nadeevo-2 carried all the loci, except for phage related, which has only been reported for the virulent strains IA-84-17975, IA-84-22113 and SD-84-15995. In contrast to the paper by A.V. Potehin et al. (2007), IL-1-DEP turned out to be highly virulent, KOMI DEP exhibited moderately virulence, and Nadeevo-2 showed no virulence at all. Interestingly, the $v t a A$ group 1 gene, the main determinant of virulence, was identified in SK-1-DEP avirulent strain and Botovo-2 and Nadeevo-2 avirulent isolates. This work contributes to a better understanding of putative virulence factors in field isolates of $H$. parasuis.

Keywords: Haemophilus parasuis, virulence factors, Glasser's disease

For successful pig industry the animals must be reliably protected against bacterial and viral pathogens, which cause serious economic damage [1, 2]. Glasser's disease is a bacterial infection typically characterized by fibrinous polyserositis, polyarthritis, meningitis, and occasionally acute pneumonia and septicemia [3]. The infection was considered to occur sporadically due to stresses. However, intensive pig farming and spreading agents which affect immune defense (e.g., circovirus type 2, swine influenza virus and porcine reproductive and respiratory syndrome virus, Mycoplasma hyopneumoniae and M. hyorhinis) are provoking mass distribution of Glasser's disease and necessitate thorough veterinary control [4].

The etiologic agent of Glasser's disease is Haemophilus parasuis, a gram- 
negative NAD (nicotinamide-adenine dinucleotide) dependent bacterium from Pasteurellaceae family [4]. H. parasuis is a common epiphyte of the upper respiratory tract of pigs, but some strains become successful in overcoming colonization resistance and causing disease [5]. Pathogen is transmitted directly from the infected sows to piglets in the first days of life, but the disease usually manifests itself 8-15 days after weaning. High genetic variability of the bacteria resulting in ability to cause pathology contributes to emergence of the virulent strains [6].

Molecular study of virulence factors has revealed differences in the genome of the virulent and avirulent strains, indicating the need for a comprehensive analysis of $H$. parasuis isolates from different countries [7]. Several authors [8-10] have identified genes encoding potential virulence factors - the outer membrane transporter (vtaA, fhuA, hhdA, hhdB, nhaC, HAPS_0254, cirA) and adhesive proteins (sclB7, sclB11). Phage genes (phage related) capable of encoding toxins are involved in the emergence of pathogenic microorganisms by a horizontal gene transfer [11].

A relationship between the biological properties of bacteria and the presence of virulence genes is not fully studied. Identification of virulence genes may be helpful in strain typing and the development of new vaccines to provide more long-term and effective protection against pathogenic strains of $H$. parasuis.

Here, genetic screening of $H$. parasuis strains and isolates, allocated to the Russian Federation from 2000 to 2011, for the presence of 10 putative virulence factors (vtaA, fhuA, hhdA, hhdB, nhaC, cirA, HAPS_0254, sclB7, sclB11 and phage_related loci) is first reported and their different combinations have been identified. In 15 of the 28 studied $H$. parasuis isolates, identified in the Russian Federation, we have found a combination of loci not described hitherto. In addition, it is shown that three avirulent strains and isolates contain vtaA group 1 gene, previously considered typical only for potentially virulent strains.

The objective of this study was to examine Russian isolates of Haemophilus parasuis for the presence of putative virulence loci.

Technique. The Haemophilus parasuis strains and isolates were as follows: type strain $H$. parasuis ATCC 19417 (serotype 4, American Type Culture Collection, Manassas, Virginia, USA); isolates AI4, SW124, NAG, D21, D95, SH6, V171, TO1, T72, NB144 (SRC Applied Microbiology and Biotechnology, Obolensk, Russia); strains IN-1-DEP, IL-1-DEP, Ural'skii-DEP, KOMI DEP and SK-1-DEP (All-Russian State Centre for Quality and Standardization of Animal Drugs and Feeds - VGNKI, Russia); isolates BEL, Botovo-2, Botovo-5, Botovo-6, Botovo-7, VN, LUB, IL2, IL3, MB, MB1, Nadeevo-2, SK3 (isolated in Russia from animals with clinical sings of Glasser's disease in 2000 to 2011 by employees of the Federal Centre for Animal Health Control). Bacto Triptone, Bacto Peptone and Difco agar (BD, Netherlands) were used for $H$. parasuis culture media. After sterilization, yeast extract (as a NAD source, $25 \mathrm{ml}$ ), horse erythrocytes $(25 \mathrm{ml})$, and fetal bovine serum $(25 \mathrm{ml})$ were added to $250 \mathrm{ml}$ medium. Bacteria were incubated at $37{ }^{\circ} \mathrm{C}$ in $5 \% \mathrm{CO}_{2}$ for $24-48$ hours.

To perform biochemical tests we used a VITEK 2 bacteriological analyzer with VITEK 2 NH ID Card (bioMérieux SA, France) for identification of fastidious microorganisms.

Bacterial DNA was isolated from pure cultures using RIBO-sorb kit (InterLabService, Moscow, Russia) according to the manufacturer's instructions.

For taxonomic attribution of NAD-dependent isolates the real-time PCR with the $H$. parasuis species-specific primers and probes designed by $\mathrm{C}$. Turni et al. [12] were used according to the reported protocol [13].

To detect putative virulence genes, 10 pairs of primers [8-10] and temperature profiles similar to those described [8-10] were used. Reaction mixture 
comprised $0.4 \mu \mathrm{mol} / 1$ of each dNTP (Fermentas, Lithuania), $0.4 \mu \mathrm{mol} / 1$ primers (Syntol ZAO, Russia), $3 \mathrm{mmol} / 1 \mathrm{MgCl}_{2}, 1 \mathrm{U}$ Taq-polymerase, 1× Green Buffer (Promega, USA) and $5 \mu \mathrm{l}$ template DNA. Amplified fragments were resolved for $15 \mathrm{~min}$ at $130 \mathrm{~V}$ on a $1.5 \%$ agarose gel containing $0.5 \mu \mathrm{g} / \mathrm{ml}$ ethidium bromide.

Statistical significance for the relationship between the presence of the gene and the virulence of reference strains, as published, was estimated by comparing correlation coefficient $\chi^{2}$ [14]. One-side Fisher's exact test was applied for calculation of the significance level achieved [14].

Results. Testing strains and isolates on biochemical characteristics showed that all the bacteria had no oxidase and urease activity, but were positive for catalase, did not produce indole and fermented glucose, galactose and mannose, that met the Burgi's criteria for $H$. parasuis [15]. All the cultures were also positive in the species-specific real-time PCR for the detection of $H$. parasuis.

Genes associated with virulence have been identified in the last few years due to suppression subtractive hybridization $[8,16]$, representational difference analysis [10] and sequencing [9, 17, 18], but a relationship between the biological characteristics of isolates and the presence of these genes is still unclear. Here, after screening for virulence-associated genes, we compared biological and molecular genetic features found in this study to those published earlier for the reference strains.

In this, 10 pairs of primers to putative virulence genes vtaA, fhuA, sclB11, nhaC, sclB7, HAPS_0254, hhdA, hhdB, cirA and phage_related were used (Table 1).

\section{Primers to putative virulence genes of Haemophilus parasuis}

\begin{tabular}{|c|c|c|c|c|}
\hline Gene & Primer & Nucleotide sequence $\left(5^{\prime} \rightarrow 3^{\prime}\right)$ & $\begin{array}{l}\text { Amplicon } \\
\text { size, bp }\end{array}$ & Author \\
\hline \multirow[t]{2}{*}{ vtaA } & YADAF1 & TTTAGGTAAAGATAAGCAAGGAAATCC & 406 & [9] \\
\hline & PADHR1 & ССАСАСААААССТАССССТССТСС & & \\
\hline \multirow{2}{*}{ fhuA } & E35-F & TCTAAGCGATGGGATTGAGC & 461 & [8] \\
\hline & E35-R & GGTGGCGTAAGACGTGATT & & \\
\hline \multirow[t]{2}{*}{ sclB11 } & A4-F & TTTGGCGTTTGATGAGTT & 186 & [8] \\
\hline & A4-R & TGGCGTTAGGTTATGGTT & & \\
\hline \multirow[t]{2}{*}{ nhaC } & E30-F & GTCCAGGAAGCATAATACA & 312 & [8] \\
\hline & E30-R & TACAAGGTGGCGAGATAA & & \\
\hline \multirow[t]{2}{*}{ sclB7 } & D32-F & CATTGGCAGAGGCTTTAT & 242 & [8] \\
\hline & D32-R & GTACGGTATTGCGGTTGG & & \\
\hline \multirow[t]{2}{*}{ HAPS_0254 } & B14-F & ACACCTTATGCTTCCGCTAT & 146 & [8] \\
\hline & B14-R & ACGGTAACAGAACAAGAGCC & & \\
\hline \multirow[t]{2}{*}{ hhd $A$} & MP_Al & GGTTCTAGTTCACAAACAGCCAATAC & 964 & [10] \\
\hline & MP_A2 & GATATTTACCCCTGCCTTCATTGTATC & & \\
\hline \multirow[t]{2}{*}{$\operatorname{hhd} B$} & MP_B1 & ATCTTGCCCTGATTAGAGAGTAGGAGT & 557 & [10] \\
\hline & MP_B2 & GTGAATATAGCCCTTATCCAAATAGGC & & \\
\hline \multirow[t]{2}{*}{ cirA } & MP_CirAl & GTATGCAGAATAAAGCCCTGCTAAAC & 215 & [10] \\
\hline & MP_CirA4 & CTGTAAAGCAATGCAATTACCGTAGTG & & \\
\hline \multirow{2}{*}{ phage_related } & Ophage 13_1 & GCTTGCGGGTAATCTGTTGT & 301 & [10] \\
\hline & Ophage13_2 & AGAATCAACCTCAGCCGAAA & & \\
\hline \multirow[t]{3}{*}{$\inf B$} & CTinfF1 & CGACTTACTTGAAGCCATTCTTCTT & 74 & [12] \\
\hline & CTinfR1 & CCGCTTGCCATACCCTCTT & & \\
\hline & CTinfP & FAM-ATCGGAAGTATTAGAATTAAGTGC-BHQ1 & & \\
\hline
\end{tabular}

All analyzed genes for putative virulence factors were found in the strains and field isolates of $H$. parasuis in various combinations (Table 2). Out of 28 strains studied, vtaA gene was detected in all strains, sclB7 and sclB11 genes were present in 24 and 25 strains respectively, and there was $\operatorname{cir} A$ gene in 18 out of 28, fhuA gene in HAPS_0254, hhdA gene in 15, hhdB gene in 14, nhaC gene in 12, and phage_related target in only 3 strains. Our findings identified 15 novel genotypes (LUB, Ural'skii-DEP, MB, SK-1-DEP, SK3, Krasnodar, 
Botovo-2, Botovo-5, Botovo-7, IL2, AI4, V171, SH6, SW124, VN) possessing unique gene combinations not described to date in publications.

\section{Putative virulence genes in studied Haemophilus parasuis strains and isolates}

\begin{tabular}{|c|c|c|c|c|c|c|c|c|c|c|c|}
\hline \multirow{2}{*}{ Strain, isolate } & \multicolumn{11}{|c|}{ Gene } \\
\hline & $v t a A$ & fhuA & sclB11 & $n h a C$ & sclB7 & $H A P S$ & 0254 & hhdA & $h h d B$ & cirA & phage related \\
\hline Type strain ATCC 19417 & + & & + & & + & & & & & & \\
\hline Strain IL-1-DEP & + & + & + & + & + & + & & + & + & + & \\
\hline Strain IN-1-DEP & + & & + & & + & & & & & & \\
\hline Strain SK-1-DEP & + & & & & + & & & & & & \\
\hline Strain Ural'skii-DEP & + & + & + & + & + & + & & & + & + & \\
\hline Strain KOMI DEP & + & + & + & + & + & + & & + & + & + & \\
\hline Isolate BEL & + & & + & & + & & & & & & \\
\hline Isolate Botovo-2 & + & + & + & & + & + & & + & + & + & + \\
\hline Isolate Botovo-5 & + & & + & & + & & & & & + & \\
\hline Isolate Botovo-6 & + & & + & & + & & & & & & \\
\hline Isolate Botovo-7 & + & + & + & + & + & + & & + & & + & \\
\hline Isolate VN & + & & + & + & + & + & & + & + & + & + \\
\hline Isolate LUB & + & & + & & + & & & & & + & \\
\hline Isolate IL2 & + & + & + & & + & + & & + & + & + & \\
\hline Isolate IL3 & + & + & + & + & + & + & & + & + & + & \\
\hline Isolate Krasnodar & + & & & & + & & & & & & \\
\hline Isolate MB & + & & + & & & & & & & & \\
\hline Isolate SK3 & + & & & & & & & & & & \\
\hline Isolate Nadeevo-2 & + & + & + & + & + & + & & + & + & + & \\
\hline Isolate AI4 & + & + & + & & + & + & & + & & + & \\
\hline Isolate D21 & + & + & + & + & + & + & & + & + & + & + \\
\hline Isolate D95 & + & & + & & + & & & & & & \\
\hline Isolate SH6 & + & + & + & + & + & & & + & + & + & \\
\hline Isolate V171 & + & & + & & & & & & & & \\
\hline Isolate TO1 & + & + & + & + & + & + & & + & + & + & \\
\hline Isolate T72 & + & + & + & + & + & + & & + & + & + & \\
\hline Isolate NB144 & + & & + & & & & & & & & \\
\hline Isolate NAG & + & + & + & + & + & + & & + & + & + & \\
\hline Isolate SW124 & + & + & + & & + & + & & + & + & + & \\
\hline In total & 29 & 15 & 26 & 12 & 25 & 15 & & 15 & 14 & 18 & 3 \\
\hline
\end{tabular}

Virulence is necessary to estimate when newly identified strains are characterized. However, expensive verification using laboratory animals still remains uncontested [19]. Thus, searching genes responsible for virulence in $H$. parasuis is an urgent task, on which many researchers are working. Genetic engineering made it possible to identify putative genes that distinguish virulent strains from avirulent [8-10]. Unfortunately, the role of these genes in the pathogenesis is unclear. Besides, the genetic screening of a larger number of $H$. parasuis isolates from different countries would make it possible to better understand the role of these genes in the symptoms associated with a particular isolate.

When studying genes encoding virulence factors in 15 reference strains of $H$. parasuis [8-10], a correlation to virulence $(\mathrm{p}<0.05)$ has been revealed for 8 genes - vtaA, fhuA, sclB11, nhaC, sclB7, HAPS_0254, hhdA and hhdB) [8$10]$. Here, we studied 5 strains and 23 isolates detected in pigs with the signs of Glasser's disease from 13 Russian regions (see Table 2), and performed screening for 10 putative virulence loci.

Locus vtaA which encodes virulence factor in $H$. parasuis is most fully characterized $[17,20,21]$. Proteins of $v t a A$-encoded family form $\beta$-folds in the outer membrane of Gram-negative bacteria [17, 20, 21]. The vtaA is involved in adhesion to host cells, counteraction to serum antibodies and avoiding phagocytosis in different bacteria $[17,20,21]$. In our findings, vta $A$ group 1 gene was detected in all 28 studied Russian strains and isolates. In contrast, A. Olvera et al. [9] identified vtaA in all reference strains with high and moderate virulence but only in a single avirulent strain 174 of serotype 7 . In validation tests the $v t a A$ gene was identified in $41.5 \%$ samples (animals' nasal swabs) sourced from the farms free of Glasser's disease, but, nevertheless, containing the H. parasuis 
genome [9, 22]. Thus, vtaA identification in all studied Russian cultures of $H$. parasuis confirms their isolation from pigs with clinical manifestation of the diseases and serves an evidence of the potential pathogenicity of these bacteria. Gene $\operatorname{cir} A$, encoding a transporter of iron, we discovered in 18 of the 28 Russian members of $H$. parasuis species. This gene was first identified by M. Sack and N. Baltes [10] as one of potential virulence-associated loci in a highly virulent Nagasaki strain (serotype 5). In total, 4 genes ( $h h d A$, hhdB, cirA and phage_related) have been identified, whose expression was confirmed in Nagasaki strain using reverse transcription PCR [10]. Later K.J. Howell et al. [23] in the study of over 200 strains of $H$. parasuis have found that cirA is one of the 10 most frequent putative virulence genes while loci $h h d A$, $h h d B$ are pseudogenes not expressed due to frameshift mutations. P. Assavacheep et al. [24] used the same primers and identified $h h d A$ in 12 of 20 samples, and $h h d B$ in 8 of 20 samples from died pigs. When screening Russian isolates, we have found $h h d A$ and $h h d B$ genes in 15 and $14 H$. parasuis strains, respectively. Unfortunately, epidemiological publications are not available for the rest of the genes.

In order to analyze a relationship of the detected loci to biological properties of $H$. parasuis we used data reported by A.V. Potekhin et al. [25] who studied the pathogenicity of strains IL-1-DEP, SK-1-DEP, KOMI DEP and isolates Krasnodar, Nadeevo-2, Botovo-2 in challenged mice, guinea pigs and piglets (Table 3).

\section{Number of challenged outbred pigs with different manifestations of Haemophilus par- asuis virulence (in vivarium) [25]}

\begin{tabular}{l|c|c|c|c}
\hline \multicolumn{1}{c}{ Strain, isolate } & Clinical signs & Dead pigs & Developed pathology & Isolation of bacterial culture \\
\hline Strain IL-1-DEP & 4 & 2 & 4 & 4 \\
Strain SK-1-DEP & 0 & 0 & 0 & 0 \\
Isolate Krasnodar & 3 & 0 & 3 & 3 \\
Strain KOMI DEP & 3 & 0 & 2 & 1 \\
Isolate Nadeevo-2 & 1 & 0 & 0 & 0 \\
Isolate Botovo-2 & 0 & 0 & 0 & 0 \\
Control & 0 & 0 & 0 & 0 \\
\hline
\end{tabular}

Six representatives of $H$. parasuis (IL-1-DEP, IL3, T72, TO1, KOMI DEP, Nadeevo-2) coincided with the high virulent reference strains IA-84-17975 (serotype 13), IA-84-22113 (serotype 14) and a moderate-virulent strain SD-8415995 (serotype 15) by the presence of 9 loci and lack of locus phage_related [810]. Despite the fact that the IL-1-DEP, KOMI DEP strains and Nadeevo-2 isolate had the same virulence factors, their biological properties varied widely [25]. Particularly, IL-1-DEP has caused the death of two of the four pigs, which indicates its high virulence. KOMI DEP infection, at moderate virulence, led to the total damage in serous membranes of the pleural, pericardial and peritoneal cavities. In animals challenged with Nadeevo-2 no clinical signs of the disease were observed.

Depression, loss of appetite and fever to $40.5-41.0{ }^{\circ} \mathrm{C}$ were reported for the first 3 days in pigs challenged with isolate Krasnodar. In these, polyserositis and loss of fibrin were also characteristic of the diseases manifestation. In this isolate pre-described as a moderate-virulent we have identified the same loci ( $v t a A$ and $s c l B 7$ ) as in a non-pathogenic strain SK-1-DEP. So we have found that genes presumably encoding virulence factors can exist both in pathogenic and non-pathogenic representatives of $H$. parasuis. For example, it appears that the avirulent isolate Botovo-2 contained 9 of 10 loci (except gene nhaC), while in avirulent strain SK-DEP-1 only 2 genes ( $v t a A$ and $s c l B 7$ ) were detected, and these loci combinations are unique and not described in publications. Overall, in more than half of the studied strains and isolates (15 of 28) we have identified 
gene combinations that do not occur, according to the published data, in none of the 15 reference strains of all 15 serotypes [8-10], which reflects the high genetic variability of bacteria [23] and indicates greater variety of genotypes than serotypes [26]. It should also be noted that in other works the putative virulence genes were examined only in 15 reference strains commonly used for serotyping, while our work is one of the few in which the genes coding for potential pathogenic factors were identified in field isolates.

Our findings support the hypothesis of multifactorial nature of virulence in $H$. parasuis, a manifestation of which depends on the genetic characteristics of the bacteria and the immunological status of the infected animal [27]. Of course, the attempted study of some potentially virulence-associated loci is not enough for a deep analysis of the problem, but it does show the need to develop a realistic model of infection based on system biology, genome sequencing and proteomic analysis in which the maximum range of isolates must be involved [23, 28-30].

Thus, all the putative virulence genes (vtaA, fhuA, hhdA, hhdB, nhaC, cirA, HAPS_0254, sclB7, sclB11 and phage_related) have been identified in 28 Russian representatives of Haemophilus parasuis in different combinations. In 15 representatives of 28 studied we have found combinations of loci which are not described to date in the literature. Interestingly, comparing our findings with data on known pathogenicity of these strains and isolates for pigs and laboratory animals indicates that IL-1-DEP, KOMI DEP and Nadeevo-2 strains, having the same gene sets characteristic of pathogenic strains of serotypes 13, 14 and 15 , showed high virulent, avirulent and moderate-virulent properties, respectively. In addition, we have shown that avirulent strains contain the vtaA group 1 gene, previously considered typical only for potentially virulent strains.

\section{R E F EREN C ES}

1. Z Z g lyadova M.Kh. Rossiiskoe predprinimatel'stvo, 2013, 14: 114-118 (in Russ.) (doi: 10.18334/rp.14.14.1444).

2. Holt k a m p D., R o t t o H., G a r c i a R. Economic cost of major health challenges in large US swine production systems - Part 1. The Pig Site, 07 May 2007. Available http://www.thepigsite.com/articles/1935/economic-cost-of-major-health-challenges-in-large-usswine-production-systemspart-1/. No date.

3. A ma no H., Shibat a M., Ka ji o N., Morozumi T. Pathologic observations of pigs intranasally inoculated with serovar 1, 4 and 5 of Haemophilus parasuis using immunoperoxidase method. J. Vet. Med. Sci., 1994, 56: 639-644 (doi: 10.1292/jvms.56.639).

4. Rapp-Gabriels o n V.J., O live i ra S.R., Pijo a n C. Haemophilus parasuis. In: Diseases of swine. B.E. Straw, J.J. Zimmerman (eds.). Ames, John Wiley \& Sons, 2013.

5. S mart N.L., M in i t s O.P., Ros e nd a l S., Fri e nd ship R.M. Glasser's disease and prevalence of subclinical infection with Haemophilus parasuis in swine in southern Ontario. Can. Vet. J., 1989, 30: 339-343.

6. Olvera A., Cerda-Cuéllar M., Aragon V. Study of the population structure of Haemophilus parasuis by multilocus sequence typing. Microbiology, 2006, 152: 3683-3690 (doi: 10.1099/mic.0.29254-0).

7. Mullins M.A., Register K.B., Brunelle B.W., Aragon V., Galofré-Mila N., B a y les D.O., J o 11 e y K.A. A curated public database for multilocus sequence typing (MLST) and analysis of Haemophilus parasuis based on an optimized typing scheme. Vet. Microbiol., 2013, 162: 899-906 (doi: 10.1016/j.vetmic.2012.11.019).

8. Wang X., Xu X., Zhang S., Guo F., Cai X., Chen H. Identification and analysis of potential virulence-associated genes in Haemophilus parasuis based on genomic subtraction. Microb. Pathogenesis, 2011, 51: 291-296 (doi: 10.1016/j.micpath.2011.06.007).

9. Olvera A., P in a S., M a ce do N., Oliveira S., Aragon V., Be nsaid A. Identification of potentially virulent strains of Haemophilus parasuis using a multiplex PCR for virulenceassociated autotransporters (vtaA). Vet. J., 2012, 191: 213-218 (doi: 10.1016/j.tvjl.2010.12.014).

10. S a c k M., B a lte s N. Identification of novel potential virulence-associated factors in Haemophilus parasuis. Vet. Microbiol., 2009, 136: 382-386 (doi: 10.1016/j.vetmic.2008.11.008).

11. B r üs s ow H., C a n c ha y a C., H a rd t W.-D. Phages and the evolution of bacterial pathogens: from genomic rearrangements to lysogenic conversion. Microbiol. Mol. Biol. Rev., 2004, 
68: 560-602 (doi: 10.1128/MMBR.68.3.560-602.2004).

12. Tu rni C., Pyke M., B l a ck a 11 P.J. Validation of a real-time PCR for Haemophilus parasuis. J. Appl. Microbiol., 2010, 108(4): 1323-1331 (doi: 10.1111/j.1365-2672.2009.04526.x).

13. Pavelko V.I., Elatkin N.P., Prut nova O.V. Vestnik veterinarii, 2013, 67: 65-68 (in Russ.).

14. M e h t a C.R., Hilto $\mathrm{n}$ J.F. Exact power of conditional and unconditional tests: going beyond the $2 \times 2$ contingency table. The American Statistician, 1993, 47(2): 91-98 (doi: $10.2307 / 2685184)$.

15. Opredelitel' bakterii Berdzhi v 2 tomakh. Tom 2 [Bergey's Manual of Determinative Bacteriology. V. 2]. Moscow, 1997 (in Russ).

16. Zhou H., Yang B., Xu F., Chen X., Wang J., Blackall P.J., Zhang P., Xia Y., $\mathrm{Z}$ h a n g J., M a R. Identification of putative virulence-associated genes of Haemophilus parasuis through suppression subtractive hybridization. Vet. Microbiol., 2010, 144: 377-383 (doi: 10.1016/j.vetmic.2010.01.023).

17. Pina S., Olve ra A., B a rcely A., B e n s a id A. Trimeric autotransporters of Haemophilus parasuis: generation of an extensive passenger domain repertoire specific for pathogenic strains. J. Bacteriol., 2009, 191: 576-587 (doi: 10.1128/JB.00703-08).

18. Olvera A., Pina S., Pérez-Simy M., O liveira S., Be nsaid A. Virulenceassociated trimeric autotransporters of Haemophilus parasuis are antigenic proteins expressed in vivo. Vet. Res., 2010, 41: 26 (doi: 10.1051/vetres/2009074).

19. Aragon V., Cerda-Cuéllar M., Fraile L., Mombarg M., Nofrarías M., Olvera A., Sibila M., S olanes D., S egalés J. Correlation between clinico-pathological outcome and typing of Haemophilus parasuis field strains. Vet. Microbiol., 2010, 142: $387-$ 393 (doi: 10.1016/j.vetmic.2009.10.025).

20. Costa-Hurtado M., B allester M., Galofré-Mila N., Darji A., Aragon V. VtaA8 and VtaA9 from Haemophilus parasuis delay phagocytosis by alveolar macrophages. Vet. Res., 2012, 43: 571 (doi: 10.1186/1297-9716-43-57).

21. Olvera A., Martínez-Moliner V., Pina-Pedrero S., Pérez-Simy M., Galofré-Mila N., Costa-Hurtado M., Aragon V., Bensaid A. Serum crossreaction among virulence-associated trimeric autotransporters (VtaA) of Haemophilus parasuis. Vet. Microbiol., 2013, 164(3-4): 387-391 (doi: 10.1016/j.vetmic.2013.02.022).

22. O live i r a S.R. Validation of a species-specific PCR able to discriminate invasive and non-invasive strains of Haemophilus parasuis. Scientific Reports, 2010: 13. Available https://goo.gl/4FLKMT. No date.

23. Howell K.J., Weinert L.A., Chaudhuri R.R., Luan S-L., Peters S.E., Corander J., Harris D., Angen Ø., Aragon V., Bensaid A., Williams o n S.M., Parkhill J., Langford P.R., Rycroft A.N., Wren B.W., Hold e n M.T., Tucker A.W., M a s e 11 D.J. The use of genome wide association methods to investigate pathogenicity, population structure and serovar in Haemophilus parasuis. BMC Genomics, 2014, 15: 1179 (doi: 10.1186/1471-2164-15-1179).

24. Assavacheep P., Assavacheep A., Turni C. Detection of a putative hemolysin operon, hhdBA, of Haemophilus parasuis from pigs with Glässer disease. J. Vet. Diagn. Invest., 2012, 24: 339-343 (doi: 10.1177/1040638711435805).

25. P ote khin A.V., Rus a le e v V.S., S hi ry a v F.A. Trudy Federal'nogo tsentra okhrany zdorov'ya zhivotnykh (Vladimir), 2007, 5: 256-263 (in Russ.).

26. Zhang J., Xu C., Guo L., Shen H., Deng X., Ke C., Ke B., Zhang B., Li A., Re n T., Li a o M. Prevalence and characterization of genotypic diversity of Haemophilus parasuis isolates from southern China. Can. J. Vet. Res., 2012, 76(3): 224-229.

27. B la ckall P.J., Turni C. Understanding the virulence of Haemophilus parasuis. Vet. J., 2013, 198(3): 549-550 (doi: 10.1016/j.tvjl.2013.09.070).

28. Zhao M., Liu X-D., Li X.Y., Chen H.B., Jin H., Zhou R., Zhu M.J., Zhao S.H. Systems infection biology: a compartmentalized immune network of pig spleen challenged with Haemophilus parasuis. BMC Genomics, 2013, 14: 46 (doi: 10.1186/1471-2164-14-46).

29. B e 11 o-Ortí B., Aragon V., Pina-Pedre ro S., Bensaid A. Genome comparison of three serovar 5 pathogenic strains of Haemophilus parasuis: insights into an evolving swine pathogen. Microbiology, 2014, 160: $1974-1984$ (doi: 10.1099/mic.0.079483-0).

30. Li J., Peng H., Xu L-G., Xie Y-Z., Xuan X-B., Ma C-X., Hu S., Chen Z-X., Y ang W., Xie Y-P., Pan Y., T a o L. Draft genome sequence of Haemophilus parasuis gx033, a serotype 4 strain isolated from the swine lower respiratory tract. Genome Announcements, 1(3): e00224-13 (doi: 10.1128/genomeA.00224-13). 